\title{
CARACTERIZACIÓN DEL RÉGIMEN DE CAUDALES EN RÍOS DE LA CUENCA DEL TAJO ATENDIENDO A CRITERIOS BIOLÓGICOS
}

\author{
D. Baeza Sanz y D. García del Jalón \\ Laboratorio de Hidrobiología. E.T.S.I. Montes, Avda. Ramiro de Maeztu s/n., 28040 Madrid, España
}

Palabras clave: Régimen fluvial, caudal mínimo, predecibilidad.

Keywords: Stream regime, minimum flow, predictable.

\author{
ABSTRACT \\ FLOW REGIME CHARACTERIZATION OF RIVERS IN THE TAGUS BASIN ACCORDING TO BIOLOGICAL CRITERIA
}

With the aim of classifying the hydrological regimes of several rivers in the Tagus basin, a study has been made of historic flow series corresponding to a sufficiently representative number of years for 16 of these rivers.

From this data several parameters of ecological interest have been calculated, which may be related with river habitability and impose habitat conditions upon their populations. An attempt has been made to find among these parameters one which describes extreme low flow conditions for long periods of several days' duration, considering this to be more biologically significant than the point flows occasionally seen in one-day periods.

On the basis of the results obtained a multivariable study, for both classification and ordering purposes, has been carried out using the classic Decorana and Twinspan programmes (HILL, 1979). This has permitted the classification of these rivers into groups with similar regime characteristics.

Subsequent analysis of the curves obtained may serve to discuss the behaviour of the rivers in minimum flow situations and the importance of basin characteristics in this behaviour. It may also serve to identify what may be the minimum maintenance flow in some rivers.

\section{INTRODUCCIÓN}

La creciente demanda social de un medio ambiente más limpio ha impuesto en la planificación hidráulica la consideración de que en los cauces regulados circulen, al menos, unos 'caudales ecológicos' o 'caudales mínimos medioambientales'. Las Confederaciones Hidrográficas y otros Órganos de Cuenca, debido a su carencia de personal con formación limnológica, ha fijado, con frecuencia, unos 'caudales ecológicos' en base a unos criterios meramente hidrológicos que nada o poco tienen que ver con la ecología.

El concepto de "caudal ecológico" comprende enfoques científicos que normalmente ocupan a profesionales diferentes, con áreas de trabajo disjuntas. El término caudal es elemento básico de hidraúlicos e ingenieros gestores del recurso agua, mientras que el adjetivo ecológico nos refiere al mundo de la biología y de la gestión de la naturaleza. Por ello, la fijación de caudales ecológicos es una tarea con una clara vocación multidisciplinar.

Un caudal circulante por un cauce podría ser considerado como ecológico, siempre que fuese capaz de mantener el funcionamiento, composición y estructura del ecosistema fluvial que ese cauce contiene.

Es evidente que existe una gama amplia de caudales circulantes que son ecológicos para un determinado cauce. Así podríamos definir, dentro de esta gama de caudales, unos extremos máximos y mínimos. En los casos más frecuentes, en que el agua es considerado un recurso escaso, nos interesará especialmente ese valor mínimo. Pero también habrá casos en que será necesario vaciar muy rápidamente un embalse (ante la amenaza de inundaciones, la necesidad de producción hidroeléctrica, o de trasvase de aguas), y en estos casos habrá que fijar también los valores máximos del caudal circulante por el cauce, para mantener la estabilidad de los recursos biológicos. 
Existe una bibliografía abundante sobre esta temática de fijación de caudales ecológicos, con diferentes criterios de base. El criterio que más coherente sería el que liga las exigencias de hábitat que tienen las especies fluviales con las variaciones de las características de éste en función de los caudales circulantes. Diversos autores han utilizado metodología basadas en este criterio, entre los que cabe señalar a TENNANT (1976), que analiza cualitativamente el hábitat piscícola en función de la hidrología de la cuenca vertiente; a WHITE (1976), que desarrolla un análisis hidraúlico entre los caudales circulantes y el perímetro mojado del cauce, asumiendo una relación creciente entre éste y la capacidad biogénica del río. Y finalmente, a STALNAKER (1979) y BOVEE (1982), quienes desarrollan un método (IFIM, Instream Flows Incremental Methodology) basado en las relaciones cuantitativas entre los caudales circulantes y los parámetros físicos e hidraúlicos que determinan el hábitat biológico.

Esta última metodología está siendo utilizada ampliamente en Norteamérica. GORE y NESTLER (1988) han presentado un análisis crítico de la misma, apuntando las líneas de investigación para su desarrollo y mejora. SOUCHON (1983) ya propuso su adaptación a los ríos franceses y GUSTARD (1987) a los del Reino Unido.

En España, hay una demanda legal de fijación de caudales ecológicos establecida en los Planes Hidrológicos, pero cuya definición no está precisada (MANTEIGA y OLMEDA, 1992). Sin embargo, también se han realizado los primeros intentos de aplicar la metodología IFIM a nuestros ríos
(GARCÍA DE JALÓN, 1990; CUBILLO et al. 1990), e iniciado el desarrollo de otras nuevas (PALAU, 1994).

Las comunidades fluviales han evolucionado sometidos a determinados tipos de regímenes de caudales y por tanto sus ciclos biológicos y requerimientos ecológicos están adaptados a las variaciones estacionales propias de dicho régimen. Así mismo, estan adaptadas a tolerar unos caudales mínimos durante un estío más o menos largo, e incluso pueden tolerar caudales muy exiguos durante uno o varios días, que obviamente no pueden mantener durante períodos largos a los que no estén adaptados.

En este trabajo se analizan una serie de regímenes de caudales en dieciseis nos distintos, mediante la elaboración de una serie de parámetros hidrológicos a los que se puede relacionar con los requerimientos ecológicos de las comunidades fluviales como predicibilidad, constancia, estabilidad y condiciones extremas por estiaje o por avenidas.

\section{METODOLOGÍA}

Se han estudiado las series históricas de caudales diarios de 16 ríos de la Cuenca del Tajo, que han sido elegidos atendiendo a que se tuvieran una serie de datos estadísticamente significativos, además de que las estaciones estuvieran por encima de regulaciones, aprovechamientos o derivaciones y que existiera una gran variedad geográfica; para poder elaborar una clasificación de los regímenes de caudales de estos ríos. En la tabla 1 se exponen los ríos con la localiza-

TABLA I. Localización geográfica y características de la cuenca de las estaciones de aforo que se han utilizado para tomar los datos del estudio. TABLE I. Geographic situation and basin charactenstics of the flow stations which have been used to collect data for the study.

\begin{tabular}{|c|c|c|c|c|c|c|}
\hline & \multicolumn{5}{|c|}{ Características geográficas de las estaciones de aforo } & \\
\hline & $\begin{array}{l}\text { Cota } \\
\text { estación } \\
\text { (m) }\end{array}$ & longitud & latitud & $\begin{array}{l}\text { superfici } \\
\text { e de la } \\
\text { cuenca } \\
\left(\mathrm{km}^{2}\right)\end{array}$ & $\begin{array}{l}\text { cota } \\
\text { máxima } \\
(\mathrm{m})\end{array}$ & Localidad \\
\hline Almonte & 318 & 5,9525 & 39,6642 & 787 & 1601 & Monroy \\
\hline Gallo & 1000 & 1,9675 & 40,8289 & 944 & 1920 & Ventosa \\
\hline Guadamejud & 772 & 2,5450 & 40,2622 & 253 & 1191 & La Peraleja \\
\hline Guadiela & 640 & 2,7853 & 40,3997 & 3342 & 1798 & Buendía \\
\hline Jerte & 256 & 6,2817 & 39,9683 & 631 & 2395 & El Torno \\
\hline Perales & 495 & 4,1347 & 40,3717 & 261 & 1461 & Villamantilla \\
\hline Tajuña & 872 & 2,6961 & 40,8217 & 658 & 1367 & Masegoso \\
\hline Tietar & 365 & 5,0444 & 40,1522 & 730 & 2188 & Arenas de San Pedro \\
\hline Trabaque & 785 & 2,3261 & 40,4261 & 361 & 1618 & Priego \\
\hline Alagon & 691 & 5,8825 & 40,5306 & 288 & 800 & Garciabuey \\
\hline Alberche & 655 & 4,5428 & 40,4278 & 1050 & 2198 & Navaluenga \\
\hline lbor & 320 & 5,5178 & 39,7722 & 266 & 1601 & Bohonal de Ibor \\
\hline Cedena & 717 & 4,5597 & 39,6358 & 53 & 1369 & Villarejo de Montalban \\
\hline Henares & 700 & 3,1200 & 40,8483 & 2597 & 2036 & Bujalaro \\
\hline Lozoya & 1210 & 3,8903 & 40,8625 & 42 & 1600 & El Paular \\
\hline Tajo & 11140 & 1,9417 & 140.6011 & 1410 & 1920 & Peralejos \\
\hline
\end{tabular}




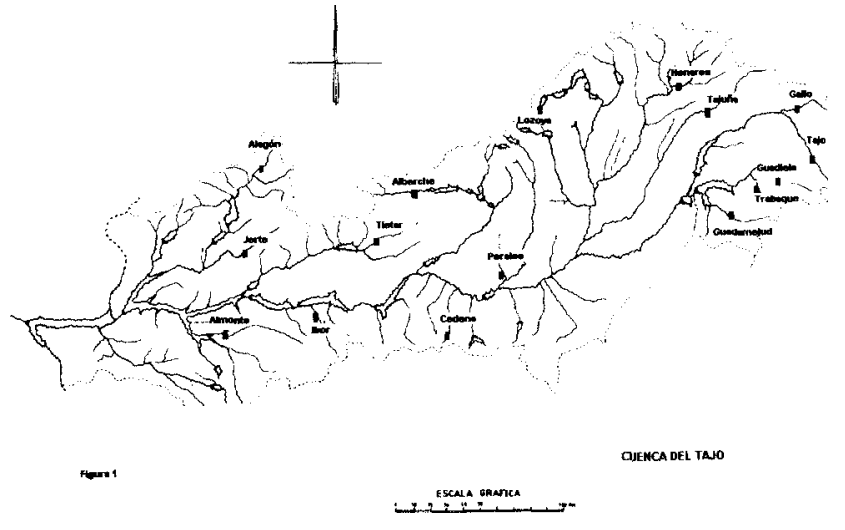

FIGURA 1. Localización geográfica de las estaciones de aforo en la cuenca del Tajo

FIGURE 1. Geographic situation of flow stations in the Tagus basin.

ción de la estación y algunas características de la cuenca y, en la figura 1 su posición en la cuenca del Tajo.

En base a estas series se han realizado el cálculo de varios parámetros de interés ecológico, basándose únicamente en métodos hidrológicos. Estos parámetros servirán para caracterizar a los ríos; y poder emparejarlos por proximidades -y ordenarlos en ejes de proximidad.

Los parámetros estudiados están fundamentados en el análisis de la variabilidad estacional que el caudal del río tiene a lo largo del año, estudiando no solamente los valores de caudales grandes y pequeños; sino también su importancia dentro de la serie hidrológica completa, la duración de estas situaciones extremas y la frecuencia con la que pueden darse.

De esta forma, podrán encontrarse valores de caudales que, de forma natural han circulado por el no, que han contribuido a personalizar al río y crearle su propia identidad, y que condicionan el nivel de desarrollo de vida acuática en cada uno de ellos.

Posteriormente se trata de encontrar una base de cálculo para establecer un posible nuevo régimen de caudales, en zonas inferiores a un aprovechamiento; en el que se conserven, en cuanto a duración, valor absoluto y frecuencia, aquellos caudales que permitan que se mantenga un nivel admisible de vida en el río, y que mantenga la identidad biológica del cauce, la ribera y el substrato ordinarios.

\section{Los parámetros}

Se han elegido parámetros que pudieran servir para su aplicación práctica, y que fuera posible particularizarlos para cada río, y conseguir que cada río tuviera su propio tratamiento especial.

El comienzo del estudio ha sido la búsqueda de un caudal bajo, al que las poblaciones fluviales hubieran estado sometidas, durante un período largo de días; este caudal tiene un gran significado biológico, ya que no es un caudal puntual, que ocasionalmente puede darse en un único día, y que puede ser soportado con mayor o menor facilidad por las comunidades; sino un caudal que ha estado mantenido durante un tiempo, y que, ha condicionado una resistencia de los individuos frente a él, durante un período grande.

Este parámetro nos va a describir las situaciones que con más frecuencia sufre la biocenosis del río, y que por lo tanto es a la que están adaptadas, huyendo de proporcionalidades fijas o números sin argumento biológico.

Posteriormente se han buscado relaciones entre este caudal y otros puntuales que no son simplemente ocasionales, sino que están relacionados con los requerimientos ecológicos de las comunidades fluviales como predicibilidad, constancia, estabilidad y condiciones extremas por avenidas o estiaje.

Para la búsqueda de este caudal, que definiremos como Q25d, se ordenaron las series diarias de caudales en orden cronológico para cada año, de estas series se calculó el caudal medio de grupos de varios días consecutivos, desde 3 días hasta 100 días, moviendo este cálculo a lo largo de toda la serie para obtener medias móviles de caudales de distinta amplitud, sobre esta segunda serie de caudales se calcula el caudal mínimo para cada grupo de días, obteniéndose una tercera serie, que corresponderá a aquellos caudales mínimos que han circulado por el río durante un grupo de días, 3, 5, 7, etc...

Una vez calculados esos mínimos por grupos de días, se repite la operación para cada año, extrayendo valores medios de todos los años estudiados, se representan gráficamente (fig. 2 ), frente a los días de los que se calcularon y se obtienen unas gráficas con pautas de crecimiento distintas, las cuales servirán para caracterizar el comportamiento del río en los momentos de estiaje. En varios de los ríos estudiados se comprueba que, hay un cambio de pendiente aproximadamente a los 25 días, por lo que usamos este caudal Q25d, como representativo, siendo el mínimo que circula por el río durante 25 días seguidos.

La variación del caudal a lo largo de los años y dentro del año, es un parámetro que influye en las comunidades fluviales en tanto que permite definir la predecibilidad del régimen, esta variación se ha estudiado dividiendo la desviación típica de los caudales medios de todos los años dividido por el módulo, parámetro denominado CVinter, y con el coeficiente de variación intraanual, que se calculó dividiendo la desviación típica de los caudales diarios de un año entre su caudal medio y luego calculando la media de este coeficiente para todos los años, este será el parámetro CVintra. 


\section{Río Tajuña}

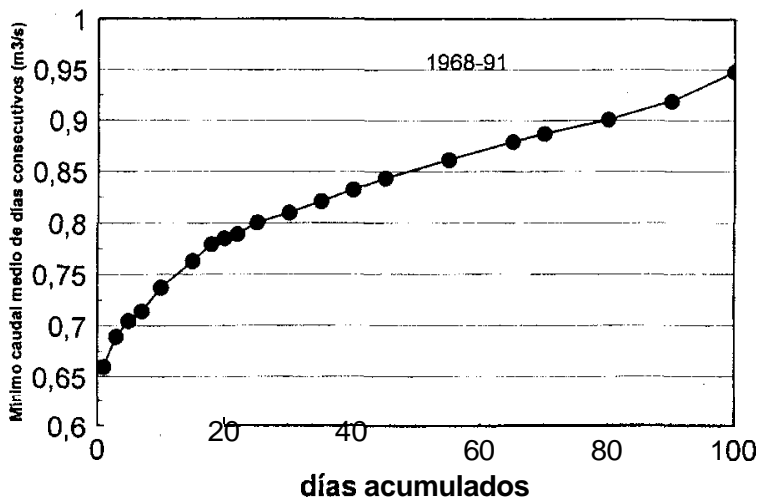

A

\section{Río Jerte}

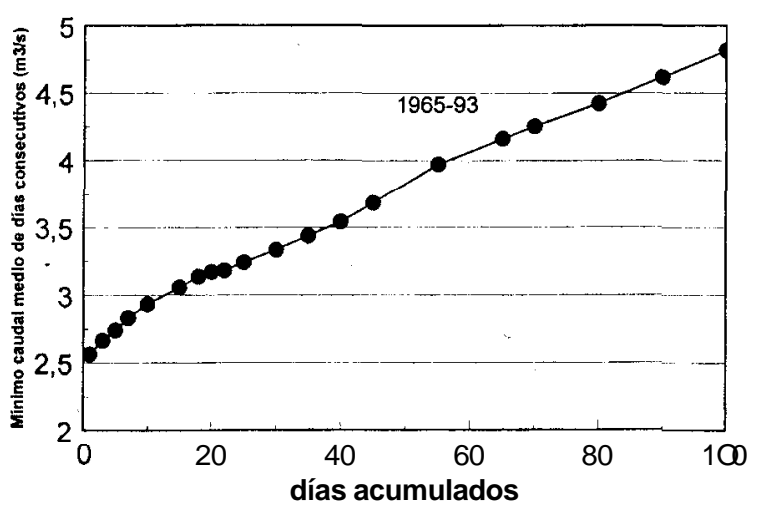

B
Río Tietar

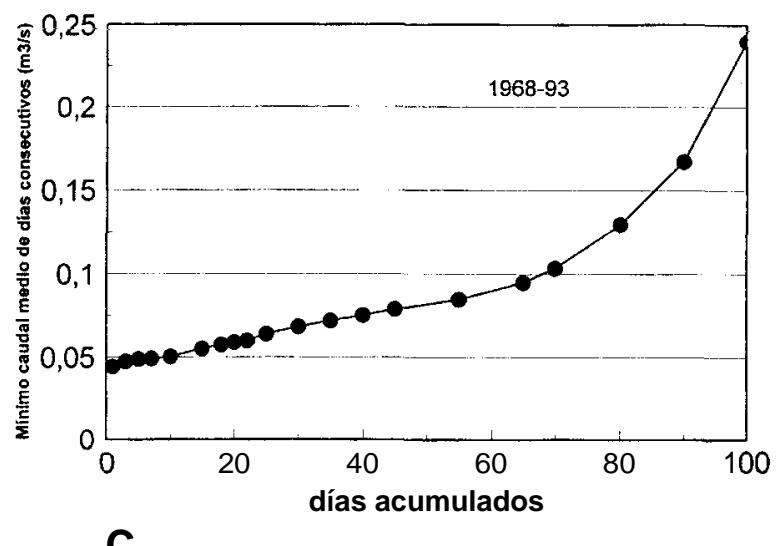

Río Cedena

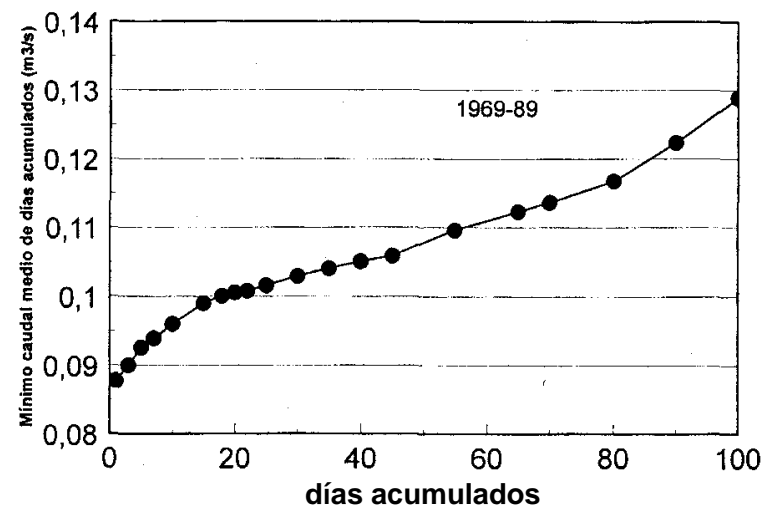

D

FIGURA 2. Representacióii de los caudales mínimo de varios días coiisecutivos variando el número de días de calculo. Se presentan cuatro tipologías de curva: la curva A, que corresponde al río Tajuña, presenta un cambio de pendiente alrededor de los 25 días y después una estabilización; en la curva B, del Jerte, no se aprecian cainbios de peiidieiite; la curva C, que corresponde al Tiétar, tiene un cambio de peiidieiite a los 70 días; y la curva D, del río Cedena, presenta dos cambios de pendiente uno a los 35 días y otro a los 80 .

FIGURE 2. Representation of the minimum flows on several coiisecutive days, varying the number of days of calculation. Four curve types are preseiited: curve A. corresponding to the river Tajuña. presents a change in slope at around 25 days, and subsequent stabilization, curve B, for the river Jerte. presents no chnnges in slope, curve C, for the river Tietar, shows a change in slope at 70 days, and curve D, for the river Cedena, presents two changes in slope, one at 35 days and another at 80 days.

Con objeto de valorar la incidencia de los mínimos mensuales en la media mensual de caudales circulantes, se ha elaborado el parámetro Qminx, como el caudal medio de caudales mínimos mensuales.

Ordenando los caudales por su magnitud a lo largo del año, desde los mayores, que raramente se alcanzan hasta los más pequeños que caracterizan la intensidad del estiaje, podemos estimar un conjunto de parámetros puntuales, de caudales que o bien son muy pocas veces superados, como el Q18, que es aquel que solamente es superado 18 días en el año, o el Q347 que es un caudal de estiaje, aquel superado durante 347 días al año. Para ver la representatividad de estos caudales extremos en las dos situaciones extremas, avenidas y estiaje, se calcularon el Q18x que es la media de los 18 caudales mayores en el año y el Q347x que es la correspondiente media de los 18 caudales más pequeños.

También esta ordenación decreciente nos sirvió para localizar el número de días que el caudal medio del río es superado, lo cual indica la persistencia de los caudales altos en el río, este parámetro lo denominamos $\mathrm{D}>\mathrm{M}$.

Para poder comparar los parámetros de unos ríos con los de otros, se normalizaron los parámetros Q25d, Q18, Q347 y 
Qminx al módulo, dividiendo estos entre el caudal medio anual de cada río. A estos parámetros se les ha denominado respectivamente Q25dm, Q18m, Q347m y Qminxm.

Con estos parámetros se pudo realizar una clasificación de los ríos, utilizando el programa estadístico Twinspan (HILL 1979b); de clasificación con lo que se ha construido un dendrograma, en el que los ríos se han agrupado en conjuntos en los que se incluyen los que tienen parámetros de valores más próximos, la separación de estos grupos se basa en un parámetro que tiene el mayor peso para producir una clasificación de primer orden, esta clasificación continua hasta obtener parejas o grupos de ríos que tienen una gran proximidad en sus regímenes de caudales.

También se hizo una ordenación en componentes principales, con el programa Decorana (HILL, 1979a), con la que se ha podido colocar a los ríos según una ordenación en dos ejes, y se ha podido comprobar también, las similitudes de sus comportamientos hidrológicos.

Los datos de aforos históricos utilizados son los publicados por la Confederación Hidrográfica del Tajo (CEDEX, 1986).

\section{RESULTADOS}

Los valores calculados de los distintos parámetros hidroló- gicos aplicados a las 16 estaciones fluviales se exponen en la tabla 2.

\section{Análisis de los parámetros hidrológicos}

El estudio de las curvas de medias móviles nos ha permitido ver distintas pautas de crecimiento en cuanto al valor de caudal, y el número de días con los que se calcula ese caudal, encontrando cuatro modelos de crecimiento:

a) Un primer modelo en el que los máximos crecimientos se producen al principio, obteniéndose un cambio de pendiente alrededor de 25 días y estabilizándose (fig. 2A); esta pauta corresponde a los ríos Tajo, Gallo, Tajuña, Guadiela, Guadalmejud y Trabaque.

b) Un segundo modelo en el que la pendiente es pequeña al principio, produciéndose un cambio brusco a una pendiente grande alrededor de 60-80 días (fig. 2B), así lo hacen el Tiétar, Almonte y Lozoya.

c) Un tercer modelo en el que no se aprecian cambios de pendientes bruscos, y la ascendencia es prácticamente lineal (fig. 2C); esto ocurre en los ríos Ibor, Alberche, Henares, Perales y Jerte.

d) Por último otros ríos presentan dos cambios de pendiente, uno alrededor de los 25 días, después una cierta estabilidad y luego otro cambio a los 80 días (fig. 2D), estos son el Cedena y el Alagón.

TABLA II. Resultados obtenidos para los parámetros hidrológicos estudiados en los 16 ríos

TABLE II. Results obtained for the hydrological parameters studied in the 16 rivers.

\begin{tabular}{|l|l|l|l|l|l|l|l|l|l||}
\hline RIOS & D>M & CVintra & CVinter & Q347m & Q18m & Q25dm & Q347xm & Q18xm & Qminxm \\
\hline Almonte & 68 & 2,24 & 0,58 & 0,01 & 5,1 & 0,005 & 0,005 & 10,8 & 0,296 \\
\hline Gallo & 142 & 0,66 & 0,46 & 0,43 & 2,1 & 0,434 & 0,395 & 3,1 & 0,692 \\
\hline Guadamejud & 80 & 1,66 & 0,66 & 0,24 & 2,5 & 0,234 & 0,223 & 4,5 & 0,000 \\
\hline Guadiela & 137 & 0,81 & 0,51 & 0,24 & 2,5 & 0,330 & 0,191 & 3,1 & 0,396 \\
\hline Jerte & 100 & 1,37 & 0,37 & 0,23 & 3,3 & 0,229 & 0,207 & 5,9 & 0,460 \\
\hline Perales & 76 & 1,91 & 0,75 & 0,02 & 5,0 & 0,016 & 0,012 & 9,0 & 0,315 \\
\hline Tajuña & 120 & 0,79 & 0,56 & 0,39 & 2,6 & 0,366 & 0,356 & 3,8 & 0,662 \\
\hline Tietar & 94 & 1,94 & 0,45 & 0,01 & 4,4 & 0,008 & 0,007 & 7,7 & 0,348 \\
\hline Trabaque & 133 & 1,24 & 0,78 & 0,27 & 2,4 & 0,265 & 0,236 & 4,4 & 0,612 \\
\hline Alagón & 82 & 2,16 & 0,68 & 0,05 & 5,0 & 0,051 & 0,041 & 8,8 & 0,297 \\
\hline Alberche & 124 & 1,16 & 0,45 & 0,07 & 2,8 & 0,169 & 0,043 & 4,8 & 0,352 \\
\hline Ibor & 90 & 1,83 & 0,61 & 0,12 & 4,0 & 0,115 & 0,100 & 7,1 & 0,506 \\
\hline Cedena & 70 & 1,63 & 0,70 & 0,21 & 3,2 & 0,188 & 0,196 & 7,2 & 0,348 \\
\hline Henares & 91 & 1,62 & 0,61 & 0,02 & 2,6 & 0,035 & 0,019 & 3,4 & 0,481 \\
\hline Lozoya & 132 & 1,04 & 0,34 & 0,11 & 3,0 & 0,106 & 0,097 & 4,1 & 0,216 \\
\hline Tajo & 99 & 1,25 & 0,47 & 0,25 & 3,4 & 0,245 & 0,046 & 0,6 & 0,500 \\
\hline \hline
\end{tabular}


Con respecto a los Coeficientes de variación, el intranual es mucho mayor que el interanual en todos los ríos, lo cual se explica ya que todos los ríos son mediterráneos, y tienen grandes oscilaciones en el año (sequias estivales y crecidas invernales). Las menores variaciones intranuales, valores menores que 1, corresponden a tres ríos, el Tajuña, Guadiela y Gallo, en los que existe algún mecanismo de amortiguación de las oscilaciones anuales (geología calcarea y cobertura vegetal). En cuanto a la interanual, son pequeñas las correspondientes a ríos montanos como el Alberche, Lozoya y Jerte; y son grandes en ríos cortos y con cabeceras en cotas bajas, como el Gualdamejud, Trabaque, Perales y Cedena.

Los valores denominados como Qminxm oscilan entre 0,21 del Lozoya y 0,69 del Gallo, los valores altos de este parámetro también nos pueden indicar ríos que tienen una regulación natural que hace que los estiajes no sean muy extremos.

El Q347/m se puede estudiar observando en que ríos el Q347 es superior al $10 \%$ del módulo y en cuales no, la primera condición la tienen el Gallo, Guadalmejud, Guadiela, Jerte, Tajuña, Trabaque, Ibor, Cedena, Lozoya y Tajo; y son menores al $10 \%$ en el Almonte, Perales, Tiétar, Alagón, Alberche y Henares; este dato sirve para confirmar la teoría de que los caudales de mantenimiento no deben sostenerse sobre procentajes fijos, sino que deben ser estudiados para cada río en particular.
Parecida interpretación se le puede dar al Q18m, en este caso podemos separar a los ríos en aquellos en los que las crecidas, considerando el q18 como un caudal que caracteriza las crecidas, son mayores de tres veces el módulo, así ocurre en el Almonte, Jerte, Perales, Tiétar, Alagón, Lozoya, Cedena, Ibor y Tajo; y son menores de tres veces, en el Gallo, Guadalmejud, Guadiela, Tajuña, Trabaque, Alberche y Cedena.

La evolución del parámetro $\mathrm{D}>\mathrm{M}$, es contraria al coeficiente de variación intraanual, en los ríos en los que los días que superan al módulo son altos; valores mayores de 100 hasta de 142, tienen una CVintra baja, y los de pocos días; hasta solamente 68 días, tienen una varianza alta, un río con pocas oscilaciones tenderá a tener los mismos días por encima del caudal medio que por debajo, aunque en estos ríos todos tienen menos días con un caudal mayor que el modulo, que los que lo tienen menor, lo que puede ser un argumento más que confirme su carácter mediterráneo.

La relación entre parámetros se estudió por correlación, y los resultados se encuentran en la tabla 3. En esta se puede ver que CVintra, está significativamente correlacionado con D > M, Q18m, Q25dm y Q347. Por el contrario CVinter está muy escasamente correlacionado con todos los demás. Las correlaciones mayores se obtienen entre Q25d y Q347.

TABLA III. Matriz de correlación entre los parámetros estudiados. se observa una gran correlación de CVintra con casi todos, excepto Qminxm; la correlación más fuerte se encuentra entre Q25d y Q347. La primera cifra corresponde al coeficiente de Pearson y la segunda a la significación estadística. Valores de $\mathrm{P}: * * * \mathrm{P}<0,01 ; * * \mathrm{P}<0,05 ;{ }^{*} \mathrm{P}<0,1$. TABLE III. Corrclation inatrix between the parameters studied, observing good correlation of CVintra with almost all, except for Qminxm. The strongest correlation was found betweeri $Q 25 d$ and $Q 347$. Values of $P ; * * P<0,005 ; * * P<0,01 ; * P<0,05$.

\begin{tabular}{|c|c|c|c|c|c|c|c|c|}
\hline Correlación & CVintra & $D>M$ & Q18/m & Q18x/m & Q25dm & Q347m & Q347xm & Qminxm \\
\hline & & & & & & & & \\
\hline CVinter & $\begin{array}{r}0,44 \\
*\end{array}$ & $-0,45$ & 0,20 & 0,36 & $-0,18$ & $-0,11$ & $-0,03$ & $-0,06$ \\
\hline CVintra & & $\begin{array}{r}-0,88 \\
* \star *\end{array}$ & $\begin{array}{r}0,84 \\
\star \star \star \star\end{array}$ & $\begin{array}{r}0,81 \\
* \star \star *\end{array}$ & $\begin{array}{r}-0,85 \\
\star \star \star \star\end{array}$ & $-0,78$ & $-0,70$ & $-0,50$ \\
\hline$D>M$ & & & $\begin{array}{r}-0,69 \\
\star \star \star \star \\
\end{array}$ & $\begin{array}{r}-0,66 \\
\star \star \star \star \\
\end{array}$ & $\begin{array}{r}0,66 \\
\star \star \star \star \\
\end{array}$ & $\begin{array}{r}0,56 \\
* * \\
\end{array}$ & $\begin{array}{r}0,52 \\
* \star\end{array}$ & 0,49 \\
\hline Q18m & & & & $\begin{array}{r}0,83 \\
* * *\end{array}$ & $\begin{array}{r}-0,77 \\
* \star *\end{array}$ & $-0,72$ & $-0,69$ & $-0,34$ \\
\hline Q18xm & & & & & $\begin{array}{r}-0,68 \\
\star \star \star \star\end{array}$ & $\begin{array}{r}-0,63 \\
* *\end{array}$ & $-0,43$ & $-0,36$ \\
\hline q25dm & & & & & & 0,97 & 0,90 & 0,50 \\
\hline Q347m & & & & & & & $\overline{0,94}$ & 0,52 \\
\hline Q347xm & & & & & & & & 0,46 \\
\hline
\end{tabular}




\section{Clasificación ríos cuenca del Tajo}

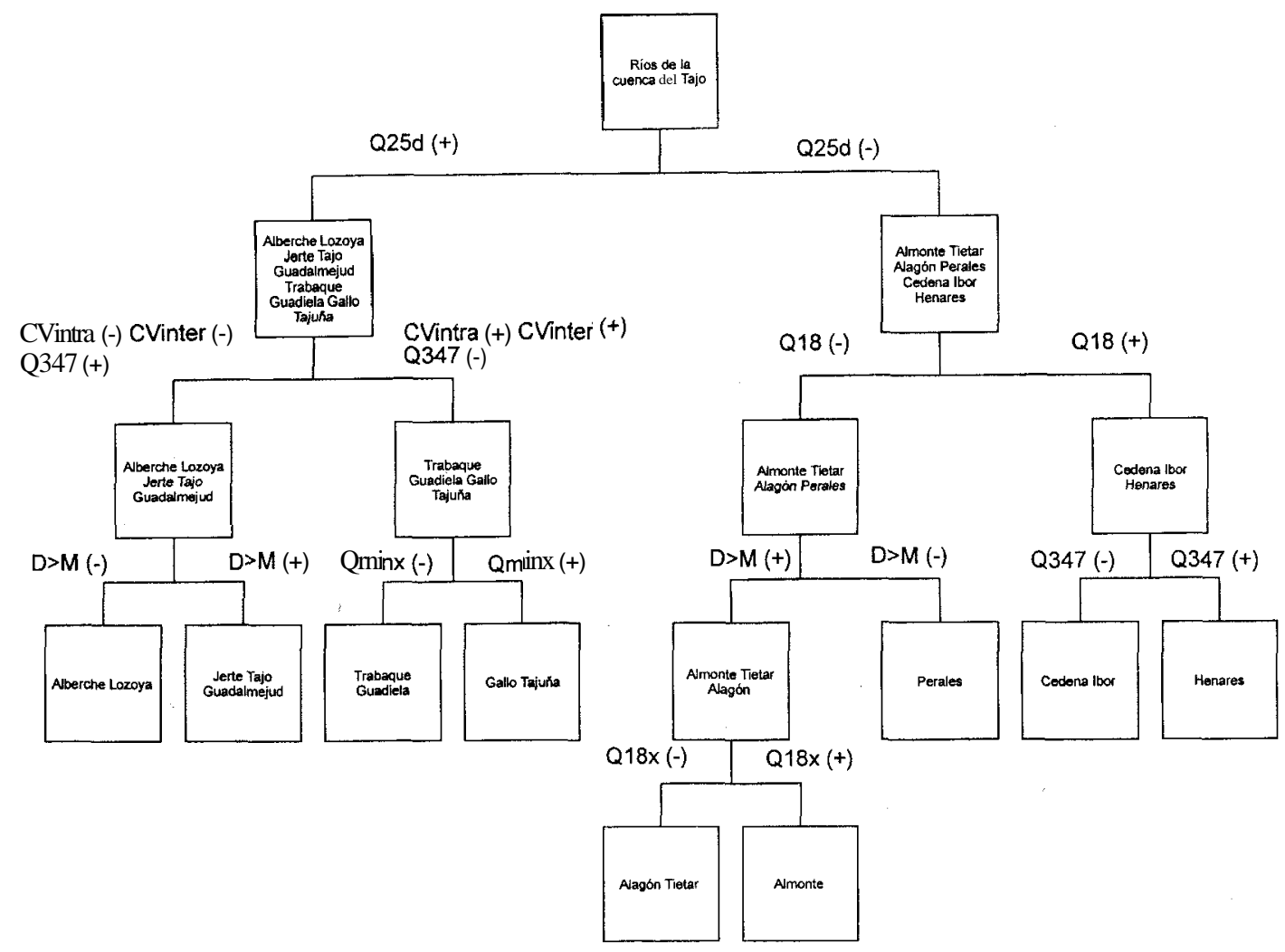

FIGURA 3. Resultados de la clasificación obtenida con TWISPAN, en la que se pueden apreciar los ríos con caracteristicas más similares y las variables que se han utilirndo para discriininar los grupos.

FIGURE 3. Results of the classification obtained with TWISPAN. showing the rivers with the most similar characteristics and the variables which have been used to discriminate the groups.

\section{Clasificación de los ríos}

En el análisis estadístico multivariante Twinspan (HILL, 1979b), cada río fue tratado como una estación de muestreo y los parámetros como especies, con el fin de clasificar por proximidades los ríos en grupos, y extraer los parámetros de mayor peso en la clasificación (figura 3 ).

\section{Ordenación}

En el análisis de ordenación, los ríos se han colocado en dos ejes: un primer eje de mayor significación, en el que los parámetros que ordenan el eje son: CVintra y Q18x como el de mayor peso en los valores positivos y un segundo eje en el que Qminx es el parámetro de mayor valor positivo y $\mathrm{D}>\mathrm{M}$ el de los negativos. El resultado de la ordenación en estos dos ejes se representa en la figura 4.

Aparecen varias parejas de ríos que están ordenadas muy cercanas tanto en el eje 1 como en el eje 2 , lo que nos indica que los valores de los parámetros de más peso en la ordenación son muy próximos. Estas parejas son: Cedena - Ibor; Tajo - Jerte, Alagón - Tiétar, Alberche - Lozoya, Trabaque Guadiela y Tajuña - Gallo.

\section{CONCLUSIONES}

Analizando los distintos tipos de regímenes y los resultados que se muestran en la tabla 2, se pueden clasificar en grupos o parejas de ríos que tengan características ecológicas similares, según los parámetros estudiados. 


\section{CLASIFICACIÓN DECORANA \\ Regímenes cuenca del Tajo}

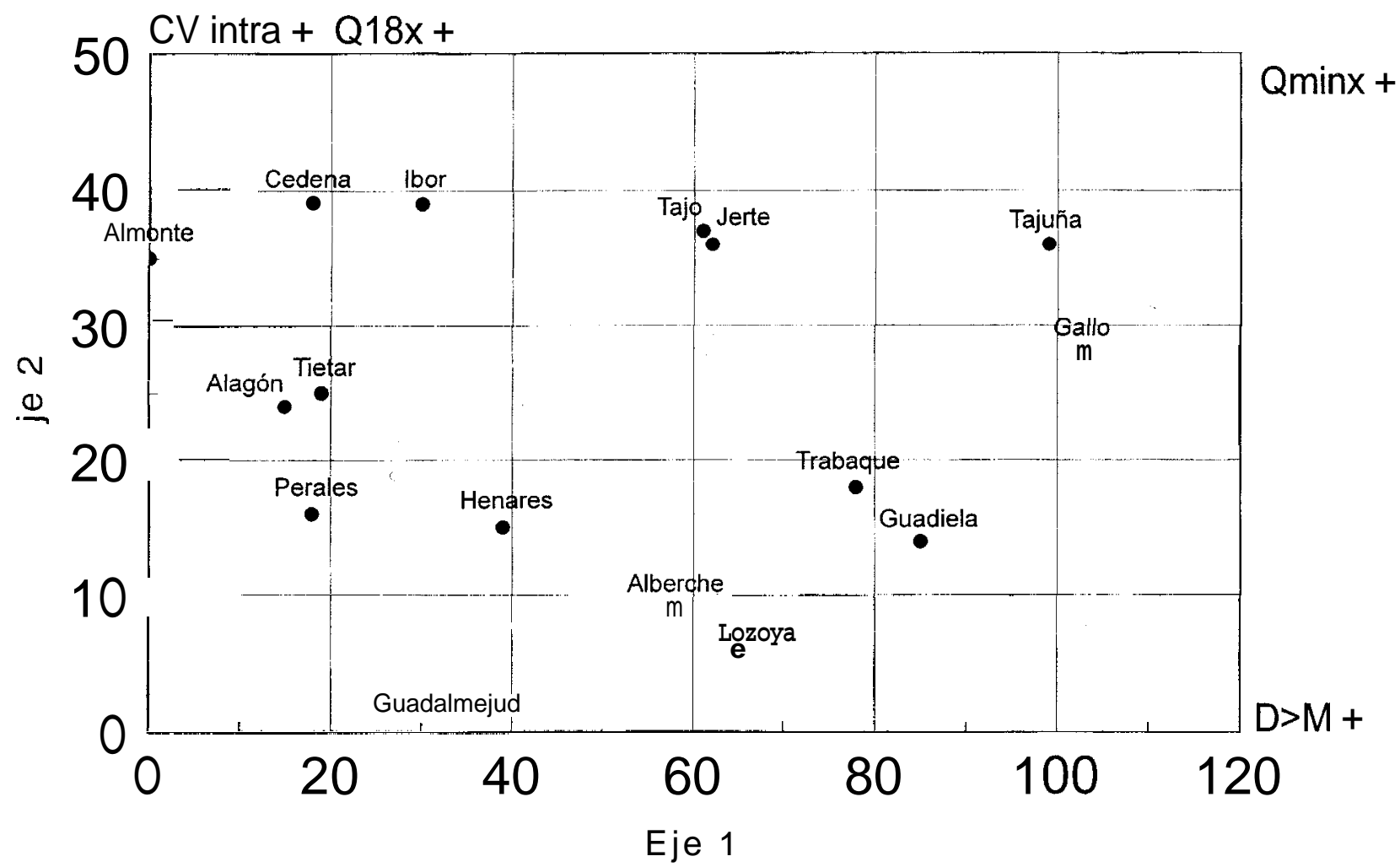

FIGURA 4. Ordenación de los ríos utilizando el programa DECOKANA, eii los dos ejes de mayor potencia; se indican sobre el riiargeii superior los paránietros que han determinado la clasificación en el eje 1 y en el margen derecho aquellos para el eje 2.

FIGURE 4. Ordering of the rivers using the DECORANA progranime, indicating in the topo margen the parameters which have deterniined classification on axis 1 and in the right margen those corresponding to axis 2 .

Los ríos con comportamiento más similar son los siguientes:

Alberche-Lozoya; estos dos ríos tienen caudales muy predecibles con una alta constancia intra e interanual. Caudales altos muy frecuentes. Sus caudales mínimos son altos y además sus estiajes son discontinuos.

Tajo-Jerte; alta estabilidad estacional. Caudales bajos altos y poco prolongados, estiajes pequeños. Regímenes predecibles. Los caudales altos son pequeños.

Tiétar-Alagón; caudales altos poco frecuentes. Poco predecibles baja constancia sobre todo dentro de un mismo año. Estiaje marcado y muy continuo, con mínimos mensuales muy bajos. Crecidas muy grandes.

Tajuña-Gallo; los caudales de estiaje son altos y poco prolongados. Gran constancia intraanual, caudal predecible. Crecidas poco importantes.
Guadiela-Trabaque; los caudales de estiaje son bajos, aunque poco prolongados en el tiempo. Gran constancia intraanual. Grandes crecidas.

Cedena-Ibor; son poco constantes entre los años, tienen caudales de estiaje bajos y prolongados y sus crecidas son fuertes.

De los demás ríos el Henares sería el más próximo a la pareja Cedena-Ibor, el Almonte el más parecido a AlagónTiétar y un poco más distinto el Perales; y por último el Guadamejud al grupo de Jerte-Tajo.

La ordenación en ejes (fig. 4), nos agrupa los ríos en dos ejes en los que tomando los ríos que se encuentran en los extremos de los dos ejes, se pueden formar cuatro grupos que están opuestos, porque son antagónicos en los parámetros que han tenido mayor peso en la clasificación; de la siguiente forma tendrán: 
CVintra, Q18x y Qminx grandes y $D>M$ pequeños.

Los ríos Cedena, Ibor, Almonte y Tiétar.

CVintra, Q18x y D > M pequeños y Qminx grandes.

Los ríos Tajo, Jerte, Tajuña y Gallo.

CVintra, Q18x y $\mathrm{D}>\mathrm{M}$ grandes y $\mathbf{Q m i n x}$ pequeños.

Los ríos Alagón, Perales, Henares, Alberche y Guadalmejud.

CVintra, Q18x y Qminx Pequeños y D>M grandes.

Los ríos Trabaque, Gúadiela y Lozoya.

Aunque en estas características, hay para algunos ríos, valores intermedios, especialmente en los parámetros $\mathrm{D}>\mathrm{M}$ y Qminx, por ser los correspondientes al segundo eje, que tiene menor potencia.

La interpretación de las curvas de medias móviles puede llevarnos a encontrar las causas de este comportamiento en algunos de los ríos estudiados.

Todos los ríos en los que se presenta una fuerte crecida del caudal al ir aumentando el número de días del cálculo, para producirse un cambio en la pendiente y una posterior estabilización de este, a partir del día 25; son ríos de una zona geográfica muy localizada, correspondiente al este de la cuenca del Tajo, y con unas características geológicas comunes.

E1 Tajo en su curso alto, y sus afluentes Tajuña, Gallo y Guadiela, además de los afluentes de este el Guadalmejud y el Trabaque, son todos ríos con este comportamiento, en los que las estaciones de aforo están situadas en zonas donde la cuenca drenada hasta ellas, es de geología caliza.

Podemos interpretar, que los mecanismos que abastecen al río, controlan la duración de los estiajes, de tal forma que los caudales muy bajos, son mantenidos durante muy pocos días, siendo situaciones de extraordinaria infrecuencia, y que inmediatamente son corregidas, por un abastecimiento no pluvial sino freático.

En este tipo de situaciones con esta tipología de curva, el caudal mínimo ecológico o de mantenimiento deberíamos buscarlo en aquella zona de la curva donde se produce la estabilidad, y nunca en la zona inicial de pendiente fuerte; por ser situaciones muy infrecuentes y que el propio funcionamiento del río soluciona, manteniéndose durante un número de días muy bajo.

Este grupo de ríos también ha coincido en los valores de varios de los parámetros estudiados, como son la predecibilidad, con una gran constancia interanual y especialmente intraanual y con unos caudales medios de mínimos mensuales altos, lo que apoya más el que son ríos con un comportamiento poco torrencial, que no presentan grandes oscilaciones.
Por el contrario los ríos Tiétar, Aimonte y Lozoya, presentan un cambio de pendiente cuando nos aproximamos a los 80 días, presentando caudales muy bajos durante períodos de tiempo largos. E1 tratamiento de estos ríos tendrá que ser opuesto a los anteriores, ya que los caudales de estiaje son muy perdurables en el tiempo, lo que posiblemente tendrá también un significado en la forma de abastecerse el propio río, donde existirá una dificultad para corregir las situaciones extremas, y por supuesto estará condicionando a las poblaciones que lo habitan.

En este caso los caudales Q25d son evidentemente muy bajos, y todos estos ríos presentan un caudal medio de mínimos mensual muy bajo, por lo que en ellos el parámetro Q25d no servirá como estimación de un caudal mínimo ecológico, sino que se tendrá que buscar en puntos de la curva pasado el cambio de pendiente, con un mayor número de días de cálculo.

Encontramos por tanto que el estudio de medias móviles puede ser una herramienta útil, para estudiar el coinportamiento del río, su relación con la geología de la cuenca y puede servir como estimador de caudales mínimos ecológicos.

\section{BIBLIOGRAFÍA}

BOVEE 1982 A Guide to Stream Hubitat Analysis usinR the Instream Flow Incremental Methodologv. Instr. Flow Inf. Paper 12. USDI Fish and Wildl. Serv. Washington. 248 pgs.

CEDEX 1986 Dutos de aforos de la cuenca del Tajo. Ministerio de obras públicas y urbanismo. Dirección General de Obras Hidraúlicas.

CHOW, V.T. 1985 Hidraúlica de los canales abiertos. Edit. Diana. México. 633 pg.

CUBILlO, F., C. CASADO y V. CASTRILLO. 1990 Estudio de Regímenes de Caudales Mínimos en los Cuuces de la Comunidad de Madrid. Agencia de Medio Ambiente. Madrid. 305 pg.

GARCÍA DE JALÓN, D. 1987 River Regulation in Spain. Reg. Rivers: Res. \& Mngt. 1, 343-348.

GARCÍA DE JALÓN, D. 1990. Técnicas hidrobiológicas para la fijación de caudales ecológicos mínimos. En: Libro homenaje al Profesor D. M. García de Viedma. 183-196. A. Ramos, A. Notario \& R. Baragaño (eds.) FUCOVASA. UPM. Madrid.

GORE, J.A. y J.M. NESTLER 1988 Instream Flows in Perspective. Rezul. Riv. Res. \& Mngt. 2, 93-102.

GUSTARD, A. 1987 A study for compensation flows in the United Kingdom. Institute of Hydrology. Wallingford. 
HILL, M.O. 1979a DECORANA a Fortran Program for Detrented Correspondence Analysis and Reciprocal Averaging. Microcomputer Power Ithaca, New York. 51 pgs.

HILL, M.O. 1979b TWINSPAN a Fortran Program for Arranging Multivariate Data in a Ordered Two-way by Classification of the Individuals and Attributes. Microcomputer Power, Ithaca, New York. 90 pgs.

MANTEIGA, L. y C. OLMEDA 1992 La regulación del caudal ecológico. Quercus, 78, 44-46.

MASACHS, $V .1948$ El régimen de los ríos peninsulares. Pub. CSIC. Instituto Lucas Mallada. Barcelona.

PALAU, A. 1994 Los mal llamados caudales "ecológicos". Bases para una propuesta de cálculo. Obra Pública $\mathrm{n}^{0} 28$ (Ríos II), 84-95.

RALEIGH, R.F., L.D. ZUCKERMAN y P.C. NELSON 1986 Habitat suitability index models and instream flow suitability curves: Brown trout. U.S. Fish Wildl. Serv. Biol. Rep. No. 82, Fort Collins. 65 pgs.

SOUCHON, F.Y. 1983 Aproche Methodológique de la Determination des Dèbits Reserves. CEMAGREF. Serv. Pêche et Hydrobiologie. Lyon.

STALNAKER, C.B. 1979 The use of habitat structure preferenda for establishing flow regimens necessary for maintenance of fish habitat. En: The Ecology of Regulated Rivers. J.V. Ward y J. Stanford. 326-337. Plennum Press.

TENNANT, D.L. 1976 Instream Flow Regimens for Fish, Wildlife, Recreation and related Environmental Resources. Procs. on Instream flow needs Symp. 326-327.

WHITE, R.G. 1976 A methodology for recommending stream resource maintenance flows for large rivers. Procs. on Instream flow needs Symp. 376-386. 